\title{
Relations débit-pertes de charges en pneumatique
}

\author{
J. P. Lecerf \\ CETIM, 52 avenue Félix Louat, 60300 Senlis
}

\begin{abstract}
L'industrie des transmissions pneumatiques n'a pas attendu la publication en 1989 de la norme ISO 6358 pour connaître les caractéristiques de débit-pertes de charges des composants qu'elle fabriquait et ces équipements ont toujours atteint les performances attendues par les utilisateurs. Alors, pourquoi ce besoin de définir les relations de débitpertes de charges en pneumatique par une nouvelle formule ? La réponse est simple : certaines évolutions technologiques nécessitent une meilleure connaissance de ces relations. C'est le cas en particulier des asservissements de position en pneumatique, domaine technologique en pleine effervescence mais ce n'est pas le seul...
\end{abstract}

\section{L LA NORME ISO 6358}

\subsection{Principe de l'essai ISO 6358}

Il s`agit de faire passer un débit d’air dans le composant pneumatique en l'alimentant en air comprimé sous une pression constante et de mesurer simultanément les pressions de l'air en amont et en aval ainsi que le débit correspondant. Afin de couvrir une plage de fonctionnement réaliste du composant, différentes pressions aval seront réglées par une vanne de réglage, de manière à répartir les points de mesures allant du débit maximal au débit minimal.

Cette méthodologie d'essai est donc fort simple. Les schémas d'un tel banc d'essai sont donnés en annexe.
Afin que les résultats de mesures soient indépendants de la réalisation technique du banc d'essai, il est imposé un certain nombre de caractéristiques dimensionnelles et opératoires.

1.2 Le dépouillement des mesures précédentes

La norme ISO décrit, pas à pas, les calculs à effectuer à partir des résultats de mesure. Ces dépouillements aboutissent à la détermination de quatre coefficients :

$$
\begin{aligned}
& C=\text { conductance sonique, } \\
& b=\text { rapport de pression critique, } \\
& A=\text { aire effective, } \\
& s=\text { coefficient d'effort de compressibilité. }
\end{aligned}
$$

1.3 Deux coefficients pour caractériser le composant

Deux coefficients, au choix, sont suffisants pour caractériser correctement les capacités en débit d'un composant pneumatique et cela, pour toutes ses conditions d'utilisation possibles.

Nous avons le choix d'utiliser l'un ou l'autre des deux jeux de coefficients ISO, soit $C$ et $b$, soit $A$ et $s$.

\section{Flow-pressure drops relationships for pneumatics}

In order to optimise the performances of the new generation of pneumatic proportional components, a good knowledge of the flow-pressure drops relationships within those components is necessary.

The ISO 6358 standard introduces two coefficients to characterize both the flow regimes of compressed air in pneumatic lines viz : subsonic flow and sonic flow. The critical pressure ratio $(P$ down/P up) for a given industrial pneumatic component depends essentially of its internal design. That ratio is of paramount importance because it determines pressure ratio for which the flow regimes change and consequently the flow-pressure drops relationships. If it is about 0.5 for correctly sized nozzles it may be only 0,15 for a real pneumatic component. This paper will emphasize the avantages and drawbacks of this ISO characterisation, and the test methods to be used to determine them experimentally. 


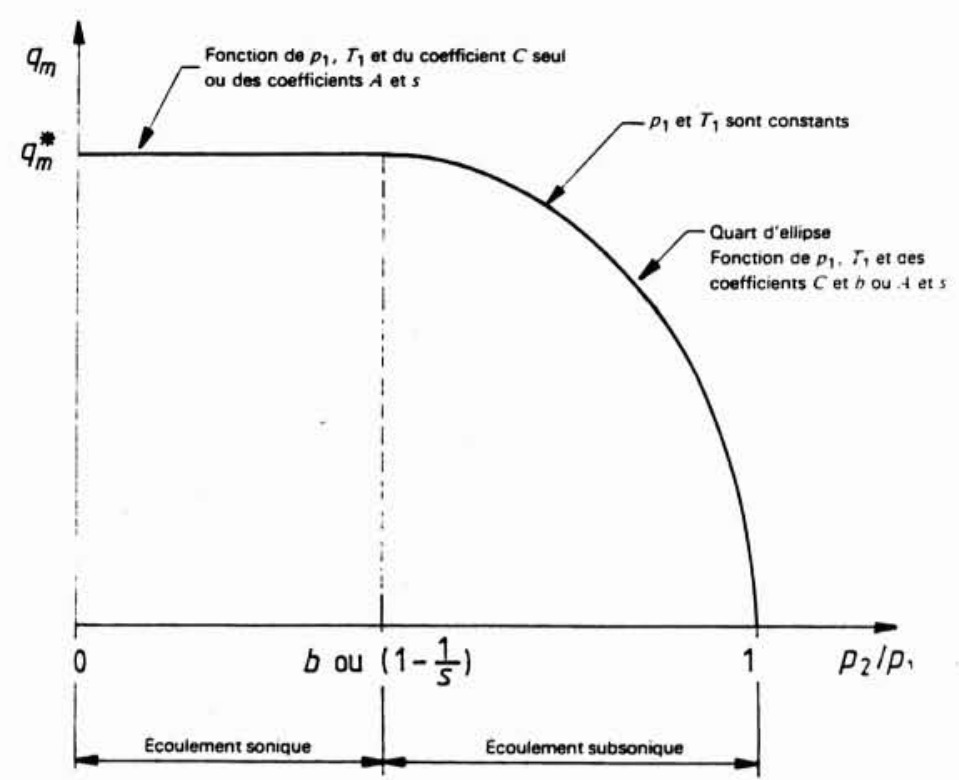

1. Courbe du débit masse en fonction du rapport des pressions absolues aval et amont.

Pour un composant dont on connaît l'un des deux jeux de coefficients, il est possible de calculer le débit masse $q_{m}$ par les relations suivantes:

\section{Equations du groupe 1}

- Pour l'écoulement sonique, c'est-à-dire pour $p_{2} / p_{1} \leqslant b$

$$
q_{m}^{*}=\mathbf{C}_{p_{1}} \rho_{0} \sqrt{\frac{T_{0}}{T_{1}}}
$$

- Pour l'écoulement subsonique, c'est-à-dire pour $p_{2} / p_{1}>b$

$$
q_{m}=\mathbf{C} p_{1} \rho_{0} \sqrt{\frac{T_{0}}{T_{1}}} \sqrt{1-\left(\frac{\frac{p_{2}}{p_{1}}-\mathbf{b}}{1-\mathbf{b}}\right)^{2}}
$$

\section{Equations du groupe 2}

- Pour l'écoulement sonique, c'est-à-dire pour $\Delta p / p_{1} \geqslant 1 / s$

$$
q_{m}^{*}=\mathbf{A} \frac{p_{1}}{\sqrt{R T_{1}}} \times \frac{1}{\sqrt{\mathbf{s}}}
$$

- Pour l'écoulement subsonique, c'est-à-dire pour $\Delta p / p_{1}<1 / s$

$$
q_{m}=\mathbf{A} \frac{p_{1}}{\sqrt{R T_{1}}} \sqrt{2 \frac{\Delta p}{p_{1}}\left(1-\mathrm{s} \frac{\Delta p}{2 p_{1}}\right)}
$$

De même, s'il est nécessaire de calculer les pressions aux bornes d'un composant pour un débit masse donné, les relations précédentes peuvent être utilisées en sens inverse.

\subsection{Pourquoi deux équations}

Deux équations sont nécessaires pour caractériser correctement les relations débit-pression lorsque le fluide est compressible alors qu'une seule équation est suffisante lorsqu'il s'agit d'un écoulement de liquide.

Cette complication apportée par les écoulements gazeux en comparaison avec les liquides est la conséquence directe des phénomènes liés à la compressibilité du gaz.

Avec un gaz s'écoulant dans un composant, nous pouvons observer deux régimes d'écoulement, l'un correspondant à un écoulement subsonique, l'autre à un écoulement sonique.

La courbe représentative du débit masse en fonction du rapport des pressions absolues aval et amont présente deux zones remarquables dont la limite correspond au rapport de pression « critique » tel que $\left(\frac{P_{2}}{P_{1}}\right)^{*}=b$.

$$
Q_{m}^{*}=C p_{1} \rho_{0} \sqrt{\frac{T_{0}}{T_{1}}}
$$

Pour un fluide donné $\left(\rho_{0}\right)$ et des conditions amont données $\left(p_{1}, T_{1}\right)$, le débit masse demeure constant quelle que soit la pression aval $p_{2}$ telle que $p_{2} \leqslant \frac{p_{1}}{b}$.

Cette particularité d'un débit limite que l'on ne peut plus augmenter en augmentant la différence de pression aux bornes du composant est spécifique aux écoulements gazeux et ne se retrouve pas avec les liquides homogènes. Certaines études ont tenté d'établir une analogie entre les régimes d'écoulements gazeux soniques et les écoulements liquides cavitants.

\subsection{Et le Kv?}

$\mathrm{Ne}$ pas confondre le $\mathrm{Kv}$ et les coefficients ISO. Avec la démarche du Kv, un seul coefficient servait à caractériser un composant pneumatique. 


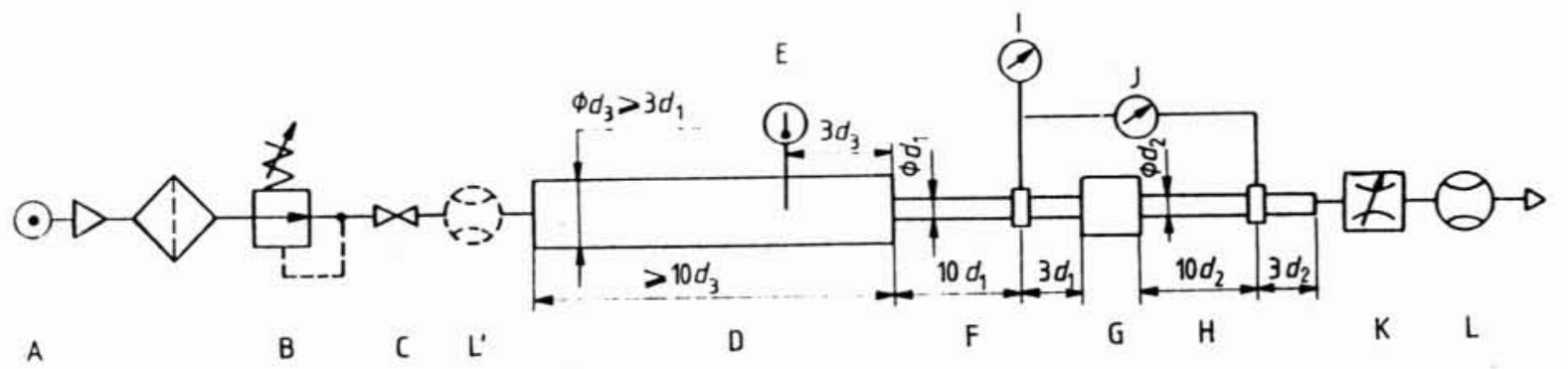

2. Circuit d'essai pour des éléments possédant des raccords d'entrée et de sortie.

Sous certaines conditions (non réalistes), l'on pourrait dire que les équations manipulées par les pneumaticiens exploitant les formules du $\mathrm{Kv}$ se ramèneraient aux formules ISO si le rapport de pression critique (coefficient ISO b) était une constante égale à 0,528 et si la température amont du fluide était toujours égale à $20^{\circ} \mathrm{C}$.

Ces conditions devraient être vraies, quels que soient les composants concernés et quelles que soient les conditions d'exploitation !

Or, le rapport de pression critique peut être proche de 0 et nous avons relevé sur notre banc d'essai pour certains composants, un coefficient ISO b inférieur à 0,1 . Quant aux températures, d'importantes variations sont observables.

\subsection{Soyons pragmatiques}

De multiples études ont été effectuées pour étudier les écoulements dans les composants pneumatiques.

Parmi les différents laboratoires ayant consacré un important effort, citons :

- Université de Bath (UK), M. Sanville [2],

- CETIM (F), M. Wartelle [1].

Tous arrivent à la même conclusion concernant les observations expérimentales.

L'écoulement de l'air dans un composant suit la courbe du débit masse donnée au paragraphe 1.4, à savoir :

- un débit masse sensiblement constant pour des rapports de pression inférieurs à la valeur critique $\left(\frac{P_{2}}{P_{1}}\right)^{*}=b$;

- un débit masse suivant une courbe sensiblement décrite par un quart d'ellipse centrée sur l'axe $\frac{P_{2}}{P_{1}}$ au point $b$.

L'ISO a retenu cette formulation mathématique.

\section{U UTILITÉ DES COEFFICIENTS ISO}

Se limiter à dire que ces coefficients permettraient de décrire au mieux les relations débit-pression d'un écoulement d'air comprimé dans un composant pneumatique n'apporterait qu'une satisfaction intellectuelle.

L'essentiel réside dans l'exploitation que l'on peut en faire, notamment aider la technologie pneumatique à progresser.

\subsection{Aide à la conception par le bureau d'études}

Maintenant que l'on peut disposer de deux coefficients différents, quels sont les enseignements que peuvent en tirer nos techniciens du BE pour concevoir un produit commercialement optimisé (coût, performances)? Parmi les questions qui se posent, l'une préoccupe particulièrement le BE : le composant a-t-il de bonnes performances ?

Les coefficients ISO permettent d'amorcer une réponse...

\section{— Le coefficient ISO A}

Il s'agit de l'aire effective de passage du fluide dans le composant pneumatique. Cette aire effective sera plus petite ou égale à la plus petite des sections d'entrée ou de sortie des composants. Plus elle sera proche de la section de raccordement, meilleure sera la performance débit du composant. Quand elle sera proche de celle du plus petit des raccordements, il ne faudra plus espérer pouvoir l'améliorer... Mais nombre de composants ne sont pas encore optimisés bien que la règle de conception consistant à dessiner une aire géométrique constante pour le passage du fluide ait été appliquée. Des zones de recirculation du fluide provoquent des réductions de passage de la veine fluide.

\section{- Le coefficient d'effet de compressibilité $s$}

Ce coefficient $s$ s'exprime en fonction du rapport de pression critique par la relation $s=\frac{1}{1-b}$.

Pour un coefficient ISO A donné, la performance en service d'un composant sera d'autant meilleure que le blocage sonique sera atteint pour la pression la plus faible, c'est-à-dire que $b \approx 0,5$.

Si $b \ll 0,5$, le composant peut être amélioré, sans changer les sections de passage, en améliorant l'écoulement du fluide à l'intérieur du composant. Un coefficient $b$ faible indique une perte d'énergie dans l'écoulement qui peut probablement être réduite.

A titre d'illustration, citons l'expérience réalisée par un technicien venu mesurer les caractéristiques de certains composants sur notre banc d'essai ; il avait un distributeur, réputé de très bonne performance. Son raccordement s'effectuait par l'intermédiaire d'une embase. Il avait le choix entre deux embases conçues selon le même critère : pas de 


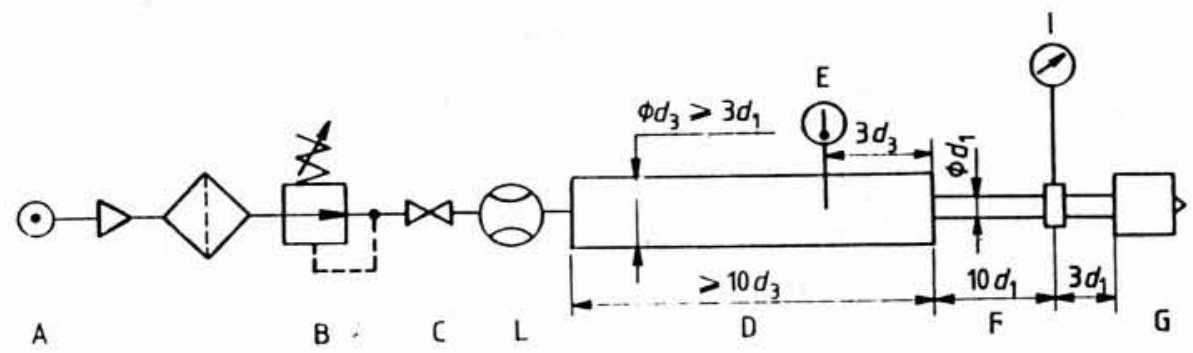

3. Circuit d'essai pour des éléments avec échappement direct à l'atmosphère.

restriction de géométrie interne, section de passage sensiblement constante. L'une était à sorties coaxiales, l'autre à sorties latérales.

Le distributeur monté avec son embase à sorties coaxiales avait d'excellentes performances, $b \approx 0,35$ et un certain coefficient ISO C.

Avec l'embase à sorties latérales, les mesures ont donné le même coefficient $C$, mais un rapport $b$ fortement dégradé, c'est-à-dire $b \approx 0,15$.

En pratique, cela signifie que le débit maximum était atteint dès 2,5 bar avec l'embase à sorties axiales, alors que le régime sonique n'était atteint que pour une pression supérieure à 6 bar avec l'embase à sorties latérales.

La méthode du Kv ne lui avait pas permis de mettre une telle différence en évidence.

\subsection{Choix de l'utilisateur}

Il est vrai que les performances en débit d'un composant ne font pas partie, généralement, des premiers critères pris en compte.

L'utilisateur avisé portera son choix sur le coefficient ISO $C$ le plus grand, dès lors que $b \geqslant 0,25$ pour une utilisation sur un réseau d'air comprimé industriel.

Mais pour que l'utilisateur puisse savoir à quelles performances il peut s'attendre avec un composant donné, il faut que le fournisseur lui communique ces caractéristiques...

\subsection{Calculs dynamiques}

L'utilisation de ces coefficients ISO est indispensable si l'on veut, par exemple, calculer le cycle d'un vérin pneumatique.

Remarquons que la pratique industrielle, forte de son expérience, sait surdimensionner de façon raisonnable un équipement pour obtenir, après réglages, les performances dynamiques d'un vérin. Mais il est bien une machine sur 10 pour laquelle la mise au point est plus délicate et nécessite des tâtonnements et des remplacements successifs de différents composants avant d'obtenir les performances dynamiques souhaitées.

Dans ce cas, un calcul aurait permis d'effectuer de précieuses économies en matériels et temps de mise au point. De même, il n'est pas certain qu'une mise au point laborieuse aboutisse à la solution optimale, notamment en coût d'exploitation pour l'utilisateur final. Car augmenter des diamètres de canalisation et de raccords, voire la taille du vérin, augmente de façon notable la consommation d'air comprimé. Gaspiller l'air comprimé n'est certainement pas une démarche industrielle à encourager.

Le calcul des cycles d'un vérin pneumatique, sous réserve que l'on connaisse les coefficients de débit ISO des différents composants, est une opération réalisable en un temps particulièrement réduit puisqu'une simulation avec le logiciel CETIM-DYNAC ne dure que de l'ordre de la minute sur un micro-ordinateur type PC.

\subsection{De la nécessité des calculs dynamiques}

Bien qu'il s'agisse de techniques récemment introduites en pneumatique, nous constatons que les asservissements pneumatiques prennent leur envol.

L'une des causes principales des difficultés rencontrées pour réaliser un asservissement pneumatique performant est la compressibilité de l'air. L'une des solutions consiste à anticiper la réponse du système et donc, à modéliser son comportement. Cette modélisation, pour être effectuée de façon satisfaisante, devra notamment prendre en compte les relations débit-perte de charges avec une précision satisfaisante. Les coefficients ISO sont alors indispensables.

Ainsi, l'équipe de chercheurs de M. Scavarda [3], Professeur à l'INSA de Lyon, exploite ces coefficients ISO pour l'étude des asservissements pneumatiques. Signalons que M. Scavarda est l'un des rares spécialistes mondiaux des asservissements pneumatiques.

\section{III $\square$ DU COÛT DE CES MESURES ISO}

La mise en pratique de cette norme par les fabricants de composants les conduirait à devoir mesurer, pour chaque voie de passage et chaque sens de passage de l'air, les coefficients définis par l'ISO 6358. Quels moyens expérimentaux seraient nécessaires?

\subsection{Le banc d'essai ISO}

Il est schématiquement décrit par la norme et dimensionnellement imposé pour ses éléments critiques. Ils ne conduisent pas, a priori, à des dépenses somptuaires.

A remarquer cependant que la moins précise des deux classes de mesure impose déjà des incertitudes limites qu'il est difficile d'atteindre avec une instrumentation industrielle. Par exemple, mesurer un débit d'air avec une incertitude relative maximale de $4 \%$ pour la valeur du débit 
Tableau 3. - Liste des éléments du circuit d'essai

\begin{tabular}{|c|c|c|c|}
\hline $\begin{array}{l}\text { Lettre de } \\
\text { référence }\end{array}$ & Paragraphe(s) & Description & Remarques \\
\hline A & $\begin{array}{c}5.3 .2 \\
6.1 .1 .2\end{array}$ & Source de gaz comprimé et filtre & \\
\hline B & - & Régulateur de pression réglable & \\
\hline C & - & Vanne d'arrêt & Préférentiellement à passage direct \\
\hline D & 5.4 & Tube de mesure de la température & \\
\hline$E$ & 一 & Instrument de mesure de la température & $\begin{array}{l}\text { Elément sensible situé sur l'axe de } \mathrm{D} \\
\text { à une distance } 3 d_{3} \text { à l'amont de } \\
\text { l'extrémité de } \mathrm{D}\end{array}$ \\
\hline $\mathrm{F}$ & 5.5 & Tube de mesure de la pression amont & \\
\hline G & - & Elément essayé & \\
\hline $\mathrm{H}$ & 5.5 & Tube de mesure de la pression aval & \\
\hline 1 & - & Instrument de mesure de la pression amont & \\
\hline$J$ & - & Instrument de mesure de différence de pression & $\begin{array}{l}\text { Lorsque } \Delta p>100 \mathrm{kPa} \text { (1 bar), } \\
\text { cet instrument peut être remplacé } \\
\text { par un instrument de mesure de la } \\
\text { pression aval }\end{array}$ \\
\hline K & 一 & Valve de contrôle de débit & $\begin{array}{l}\text { Doit avoir un débit supérieur à celui de } \\
\text { l'élément essayé }\end{array}$ \\
\hline L & - & Instrument de mesure de débit & $\begin{array}{l}\text { Peut aussi être placé en L'à l'amont } \\
\text { de D }\end{array}$ \\
\hline
\end{tabular}

mesuré conduit à devoir utiliser plusieurs débitmètres pour couvrir la plage des débits nécessaires pour mesurer les coefficients ISO d'une voie de passage.

Cette norme impose aux fabricants de devoir s'équiper d'une instrumentation de mesure performante... et encore coûteuse.

\subsection{Le dépouillement des mesures}

Il est soigneusement décrit dans la norme et un dépouillement manuel est envisageable... au prix d'un temps important. L'automatisation des calculs est plus que souhaitable. D'autant plus que pour conduire correctement l'essai, il est souhaitable d'effectuer les dépouillements en « temps réel » afin de déceler toute anomalie éventuelle du comportement du composant.

Cette norme incite fortement les fabricants à devoir utiliser une chaîne d'acquisition de données couplée à une unité de calculs, par exemple un ordinateur.

\subsection{La salle des compresseurs}

La norme impose de maintenir un débit constant dans chaque voie du composant. Cela suppose donc que le fabricant soit capable de faire passer en continu le maximum de débit dans chacun de ses composants, y compris les plus gros... Cela conduirait à avoir des compresseurs surdimensionnés en débit par rapport aux autres utilisations courantes de l'entreprise.

Cette norme est difficilement applicable par les fabricants de façon économique, pour les composants de fort débit.

\subsection{Une alternative à la méthode ISO 6358}

A la demande de la profession pneumatique, le CETIM a recherché une méthode d'essais qui permettrait la mesure des coefficients ISO avec des équipements plus économiques.

Le premier objectif a été de pouvoir caractériser les composants de grande dimension avec une capacité en débit raisonnable pour les compresseurs. Le principe de la vidange d'un réservoir d'air comprimé a été retenu.

Le deuxième objectif a été de contourner la difficulté des mesures directes de débits instationnaires en effectuant des mesures de température de l'air comprimé.

Cette étude réalisée au CETIM par M. Benchabane [4 et 5], en collaboration avec M. Bonis, Directeur de thèse à I'UTC (Université de Technologie de Compiègne) a permis de mettre au point une méthode de mesure des coefficients de débit définis par l'ISO 6358. Ces travaux ont fait l'objet d'un mémoire de thèse disponible à l'UTC et au CETIM.

\section{IV — DES BESOINS INDUSTRIELS}

Bien que publiée en 1989, cette norme n'est pas devenue une pratique systématique pour l'ensemble de la profession. Les contraintes économiques qu'elle entraîne en sont probablement l'une des causes majeures. Bien que la méthode par décharge de réservoir permette de réduire l'importance de ce handicap, d'autres bonnes raisons demeureront.

4.1 Les équipements pneumatiques à hautes performances dynamiques

Parmi ces équipements, citons les axes pneumatiques. La caractérisation des constituants avec les coefficients 
ISO 6358 est pratiquement incontournable. De même, si l'on veut calculer correctement le temps de réponse d'un vérin pneumatique travaillant en butée mécanique, la connaissance des coefficients ISO est indispensable.

Pour toutes les applications où un calcul de simulation de process est nécessaire, la connaissance des coefficients ISO s'impose.

\subsection{Les réseaux de distribution de l'air comprimé}

S'agissant des réseaux de canalisations reliant la salle des compresseurs ( 8 bars) jusqu'au poste de travail ( 6 bars), une caractérisation des composants par des coefficients de débit pour des régimes d'écoulement qui ne correspondent pas à leur utilisation normale est inutile. Ces composants seront toujours utilisés avec des écoulements faiblement subsoniques. Une caractérisation de leur comportement avec un seul coefficient de débit est industriellement acceptable. Rappelons que les installateurs ont l'habitude de travailler avec la notion de longueur équivalente, qui présente une utilisation aisée pour le dimensionnement d'une installation directement par les installateurs. De simples additions sont nécessaires. Envisager de contraindre la profession des installateurs à dimensionner leurs réseaux de distribution d'air comprimé à partir des coefficients ISO sans l'aide d'un calculateur spécialisé ne serait vraiment pas raisonnable.

\subsection{Les équipements d'automatisation pneumatiques courants}

Lorsque la performance dynamique n'est pas difficile à obtenir, eu égard à l'expérience acquise, les calculs précis que permettent les coefficients ISO ne se justifient pas. Il faut reconnaître que tant que les équipements pneumatiques seront systématiquement surdimensionnés par rapport à l'usage qui en est fait, les calculs précis de dimensionnement demeureront exceptionnellement indispensables. A noter cependant qu'une tendance à réduire le surdimensionnement systématique se fait jour...

\section{$\mathrm{V} \square \mathrm{CONCLUSIONS}$}

L'évolution technologique des composants pneumatiques est orientée vers une amélioration des performances des technologies existantes et le développement de nouveaux composants de type proportionnel. Cette évolution n'est possible qu'avec l'aide de calculs d'optimisation dans lesquels les relations de débit-pertes de charges deviennent déterminantes. La norme ISO 6358 permettant une approche plus précise de ces relations est devenue un outil de travail incontournable, et cela, malgré son coût de mise en œuvre. Des méthodes alternatives réduisant le coût de mise en œuvre de l'ISO ont été développées.

\section{Bibliographie}

[1] WARTElle C. - Caractéristiques de débit des appareils à fluides compressibles. Les mémoires techniques du CETIM, $\mathrm{n}^{\circ} 13,1972$.

[2] SANVILLE F.E. - New methode of specifying the flow capacity of pneumatic fluid power valves. 2nd Fluid Power Symposium, 1971.

[3] Transmissions pneumatiques éléments traversés par un fluide compressible. Détermination des caractéristiques de débit. norme ISO 6358, 1989.

[4] Benchabane S. - Coefficients de débit pneumatiques ISO 6358. Méthode de mesure à vocation industrielle. Rapport CETIM n $175060,1991$.

[5] Benchabane S. - Méthode de mesure à vocation industrielle. Vidange des capacités pneumatiques. Rapport CETIM $\mathrm{n}^{\circ} 176060,1992$. 